\title{
Acute intestinal necrosis secondary to idiopathic intimal hyperplasia of visceral small arteries: A case report
}

\author{
Kamrani ${ }^{1 *}$, Sohn JD ${ }^{1}$, Tchomobe $\mathrm{G}^{2}$, Stelter $\mathrm{N}^{2}$, Zhong $\mathrm{N}^{3}$, Boyan $\mathrm{W}^{1}$ and Clarke $\mathrm{KO}^{1}$ \\ ${ }^{1}$ Department of Surgery, Newark Beth Israel Medical Center, Newark, NJ, USA \\ ${ }^{2}$ School of Medicine, St. George's University, West Indies, Grenada \\ ${ }^{3}$ Department of Surgery, Larkin Community Hospital, Miami, FL, USA
}

\begin{abstract}
Intestinal ischemia is caused by a reduction in blood flow to a level that is insufficient for the delivery of oxygen and nutrients required for cellular metabolism. It is often related to acute arterial occlusion (embolic or thrombotic), venous thrombosis, or hypoperfusion of the mesenteric vasculature causing a non-occlusive ischemia or vasoconstriction. Acute intestinal ischemia due to severe hyperplasia of the intima of mesocolic/mesenteric small arteries consistent with fibromuscular dysplasia (FMD) has not been previously described. We report the case of a 47-year-old otherwise healthy male who presented with an acute abdomen with negative computed tomography angiogram but ischemic enterocolitis with necrosis due to severe intimal thickening of the mesenteric/mesocolic small arteries resembling the pattern of FMD.
\end{abstract}

\section{Introduction}

Fibromuscular dysplasia (FMD) is an uncommon, noninflammatory vasculopathy involving primarily large and mediumsized muscular arteries. FMD commonly affects renal, carotid, vertebral, and coronary arteries $[1,2]$. The disease may occur at any age, but it most commonly presents in early to middle adulthood, often affecting women of childbearing age more commonly than men [3]. The higher female prevalence has led many to believe a hormonal influence in the development of FMD, but the etiology is unknown. FMD is categorized into intimal, medial and adventitial types and classified as local and multifocal disease [4].

Here we report a case of ischemic necrosis of the small and large bowel secondary to severe luminal stenosis from intimal hyperplasia of small mesenteric/mesocolic arteries. The patient presented in this case report did not have other vasculopathies, recent myocardial infarction, atherosclerotic events or any other identifiable risk factors associated with FMD.

\section{Case report}

The patient is a 47 -year-old Hispanic male with no significant past medical history except for a laparoscopic cholecystectomy a year prior to presentation who presented with intractable, constant severe acute abdominal pain associated with several episodes of watery, nonbloody diarrhea and non-bloody, non-bilious vomiting. The patient denied smoking, alcohol or recreational drug use. Blood pressure and heart rate were within normal limits, but the physical examination was significant for diffuse abdominal tenderness with involuntary guarding and rebound. Initial laboratory values revealed leukocyte count of $34,000 \mathrm{~mm} 3$, lactic acid $5.2 \mathrm{mmol} / \mathrm{L}$, platelet count of $90,000 \mathrm{~mm} 3$, hemoglobin $17.1 \mathrm{~g} / \mathrm{dL}$, glucose $190 \mathrm{mg} / \mathrm{dL}$, slightly elevated AST 49 U/L, ALT $57 \mathrm{U} / \mathrm{L}$, glucosuria 2+ and ketonuria 2+. Urine toxicology was negative. Intravenous resuscitation, pain management, and empiric broad-spectrum antibiotics were initiated immediately.
An abdominal computed tomography angiography (CTA) was obtained for the purpose to evaluate mesenteric ischemia. After careful review, no evidence of thrombosis or filling defect were identified in the celiac, superior mesenteric artery (SMA) and inferior mesenteric artery (IMA). In addition, there was no evidence of pneumatosis or portal venous gas. Incidentally, the patient was found to have a replaced right hepatic artery off the SMA as well as diverticulosis without evidence of diverticulitis (Figure 1).

Despite aggressive resuscitation, the patient deteriorated within the first 12 hours after admission. As a result, he was taken emergently for an exploratory laparotomy. The external appearance of the small bowel was dusky and gray in its entirety from the ligament of Treitz to the ileocecal valve (Figure 2). The mesentery of the small bowel, however, appeared healthy with appropriate capillary refill with strong Doppler signals over large mesenteric vessels (Figures 3 and 5). The serosa of the large bowel also appeared dusky along the ascending, transverse and descending colon. The mesentery of the small bowel and large bowel was investigated with indocyanine green angiography. The proximal mesenteric vessels were patent, perfusing well toward the distal vessels which revealed no flow (Figure 4). With these intraoperative findings, the decision was made to leave the abdomen open, continue resuscitation, and plan for a second look.

A second look laparotomy was performed the next day after resuscitation, with no change in the appearance of the hypoperfused small bowel, but the entire colon and distal portion of the terminal

*Correspondence to: Kambiz Kamrani, MD, Department of Surgery, Newark Beth Israel Medical Center, 201 Lyons Newark, NJ 07712, USA, E-mail: kambiz@ gmail.com

Key words: acute intestinal necrosis, idiopathic intimal hyperplasia, fibromuscular dysplasia

Received: December 01, 2018; Accepted: December 10, 2018; Published: December 13, 2018 


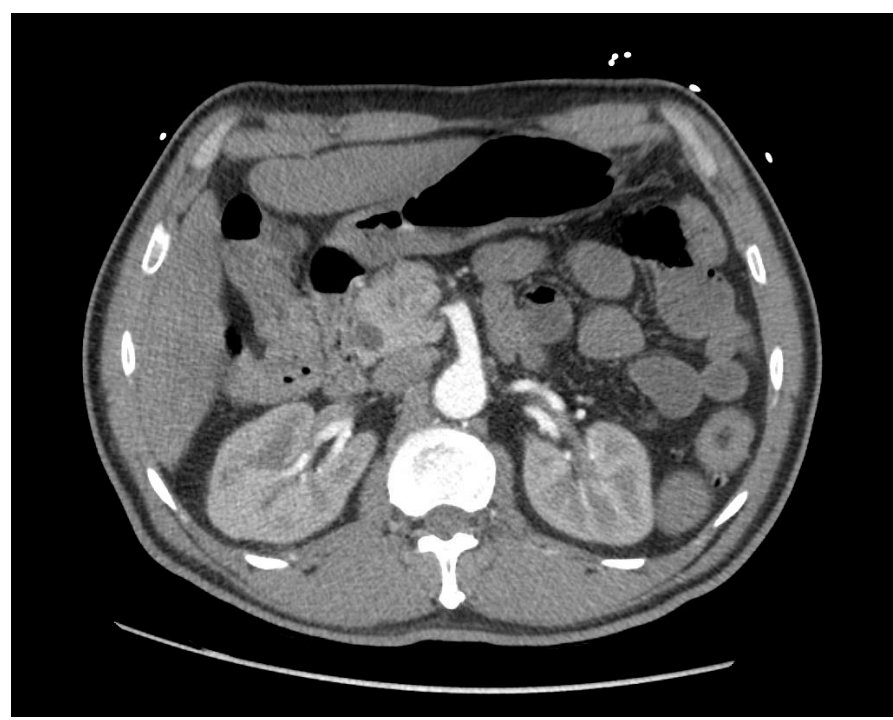

Figure 1. Computed tomography angiography of the abdomen documented patient superior mesenteric artery without evidence of pneumatosis

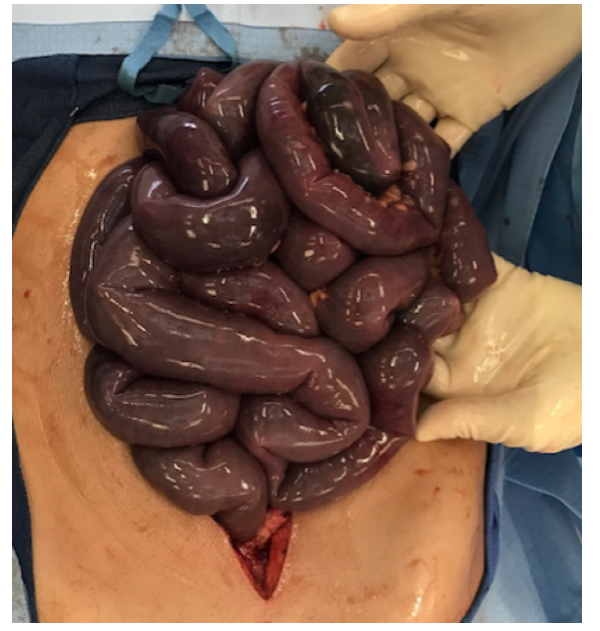

Figure 2. Appearance of ischemic small bowel of first laparotomy

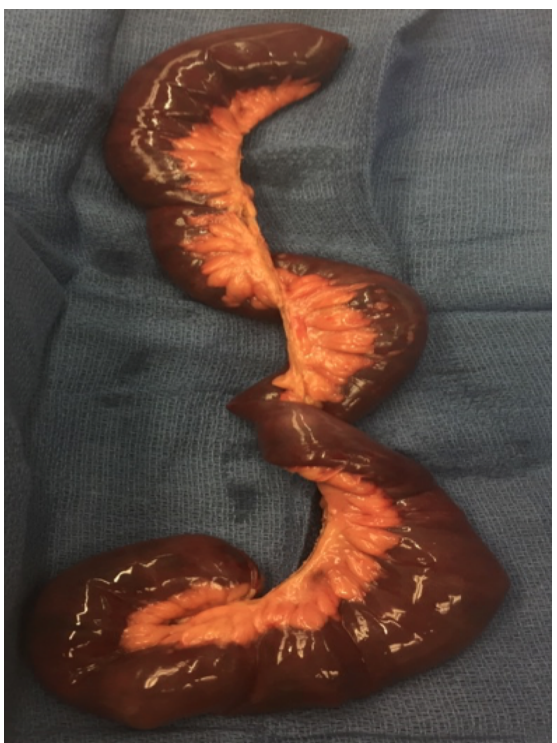

Figure 3. Viable mesentery with resected ischemic small bowel

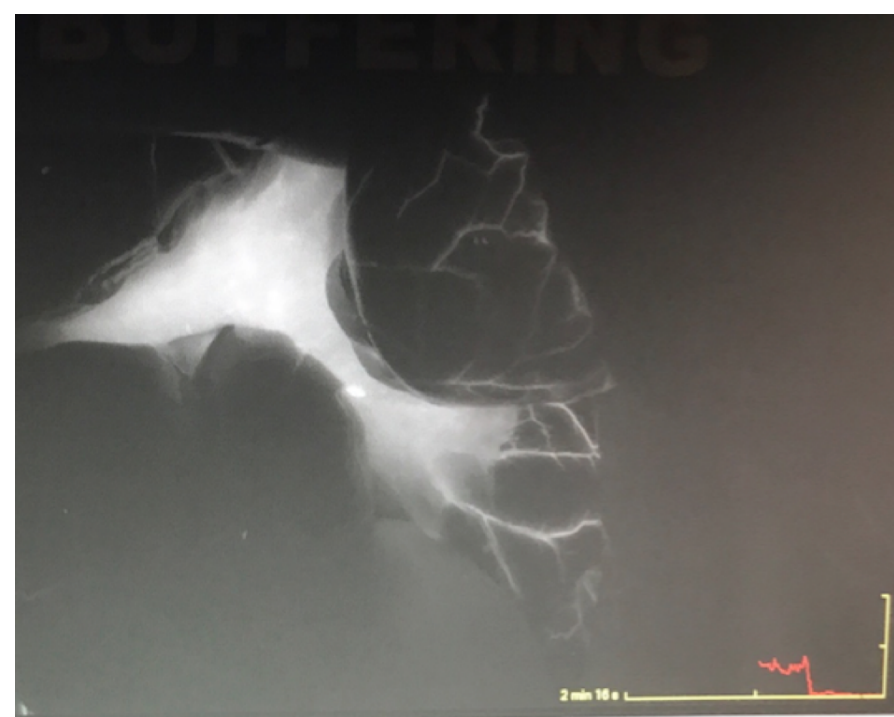

Figure 4. Intraoperative indocyanine green angiography on first laparotomy showing patent mesenteric large vessels with no flow to distal mesenteric small vessels

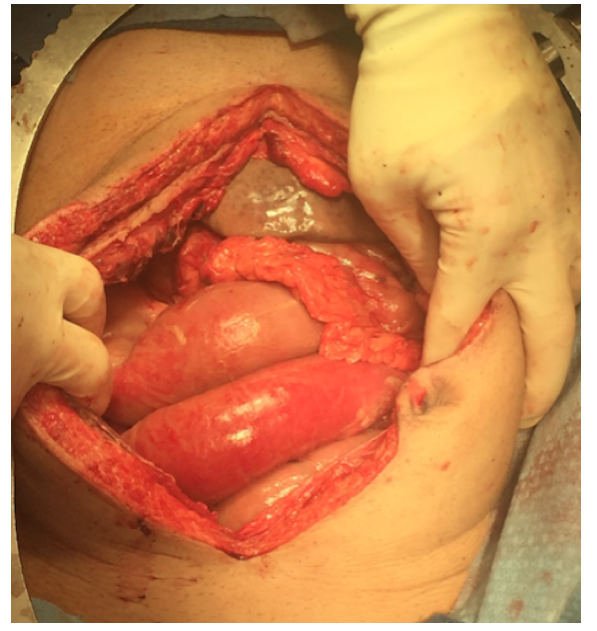

Figure 5. Healthy, viable mesentery upon small bowel resection on second laparotomy

ileum was necrotic. Additionally, the right lobe of the liver was ischemic in appearance attesting to the replaced right hepatic artery off the SMA. A second indocyanine green angiography exam was performed revealing no distal circulation with proximal mesenteric vessels appearing patent and well perfused (Figure 6). A small bowel resection, leaving approximately $80 \mathrm{~cm}$ of small bowel, with a subtotal colectomy to include all areas of bowel with evidence of necrosis was performed and the patient was left in intestinal discontinuity.

Over the course of months, the patient returned to the operating room for serial resections of the remaining portions of compromised small bowel with ileal-rectal anastomosis. He then developed an enterocutaneous fistula and wound dehiscence. The patient was sustained with parenteral nutrition for his resultant short bowel syndrome. Ultimately, after repeated bouts of sepsis, the patient and his family decided to be placed on hospice and comfort care.

The total length of resected small bowel was $343 \mathrm{~cm}$ in length and 7 to $8.5 \mathrm{~cm}$ in diameter. Histological evaluation of the small bowel demonstrated was ischemic necrosis accompanied by intimal thickening of the small mesenteric arteries with $90 \%$ luminal stenosis (Figure 7). The cecum, ascending, transverse and descending colon 
were found to be $158 \mathrm{~cm}$ in length and $6 \mathrm{~cm}$ in diameter with gross brown discoloration. Similarly, histological analysis demonstrated ischemic necrosis and marked intimal thickening of the mesocolic small arteries with 90\% luminal stenosis (Figure 8).

\section{Discussion}

The patient presented is a 47 -year-old Hispanic man with a past surgical history of a cholecystectomy and no other contributory history, suffering from acute onset diffuse small and large bowel ischemic necrosis as well as partial liver ischemia involving the right lobe of the liver.

FMD is a non-inflammatory, non-atherosclerotic disease process resulting in arterial stenosis. Most commonly seen in the renal (75-80\%) and carotid arteries of middle-aged individuals, FMD is rarely seen in the vertebral and external iliac arteries [1-3]. While the underlying etiology is poorly understood, it is believed to be under the influence of hormonal control, as it is seen most often in women of childbearing age. Complications of FMD can include complete arterial occlusion, arterial dissection, and arterial aneurysm and rupture, all of which can

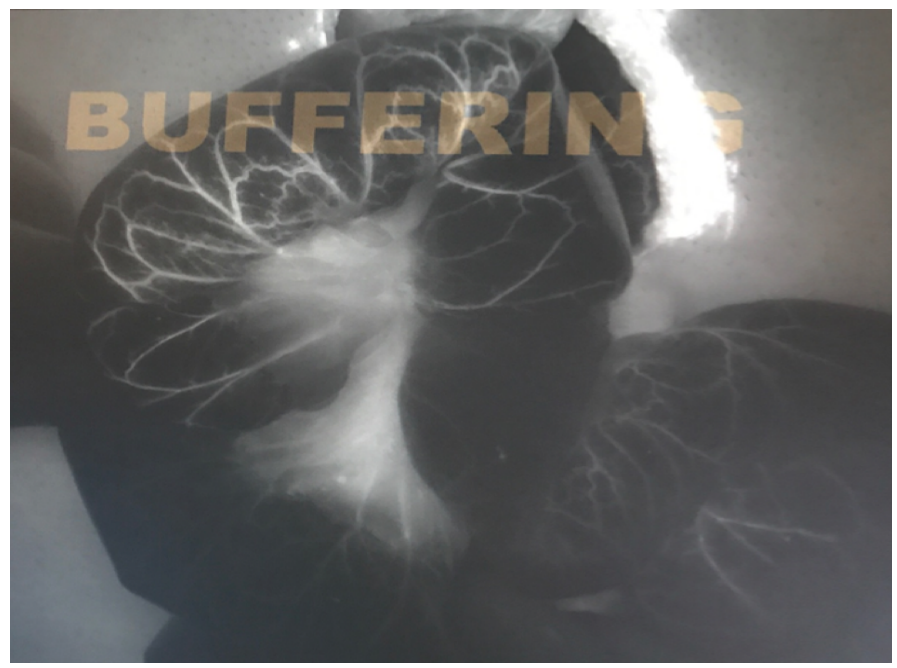

Figure 6. Intraoperative indocyanine green angiography on second laparotomy showing patent mesenteric large vessels with no flow to distal mesenteric small vessels

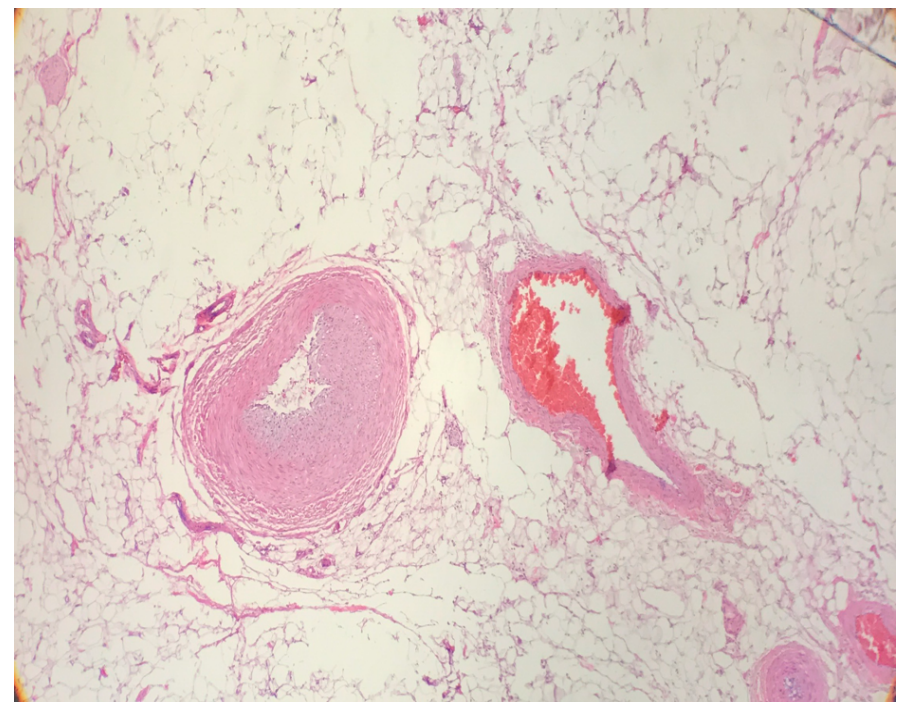

Figure 7. Luminal narrowing of mesenteric distal vasculature with $90 \%$ luminal stenosis, utilizing hematoxylin and eosin stains

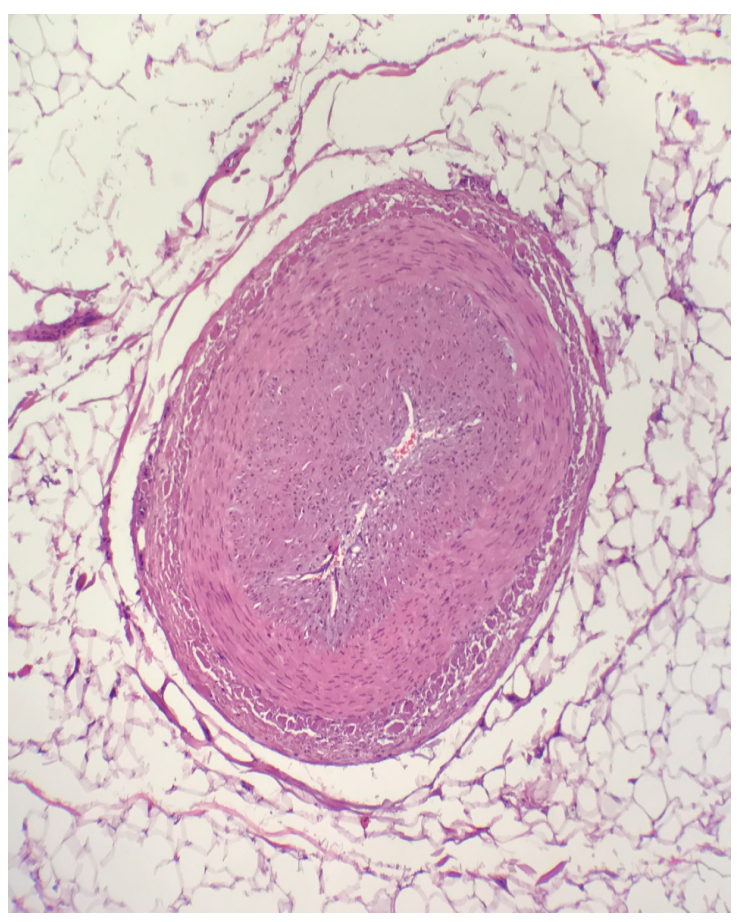

Figure 8. Luminal narrowing of mesocolic bowel distal vasculature with $90 \%$ luminal stenosis, utilizing hematoxylin and eosin stains

lead to hypertension, end-organ damage, and in severe cases, death, as this disease process is often chronic in nature [3]. A lack of chronic symptoms in this case confounded the typical presentation of FMD.

As mentioned above, FMD is associated with medium sized arteries. CT angiography is the best test to diagnose FMD with decreasing sensitivity in disease affecting smaller branched arteries. This case of FMD is atypical due to the diffuse nature of mesenteric terminal end arterial involvement and the disease's isolation to the mesentery. In addition, intimal fibroplasia, the pathological process of collagen deposition within the intima, as seen in this patient, represents only $10 \%$ of all FMD diagnosis with the other distributions primarily involving the media of the vasculature [4]. Based on our literature review, this is yet a further unique presentation that has not been documented.

The initial management of FMD includes anticoagulation or antiplatelet therapy and percutaneous balloon angioplasty in patients with chronic progressive symptoms. Due to the acute onset and rapid progression of our case and the nature of the disease being located primarily in the terminal arterial branches of the mesenteric arteries, the typical management of FMD was not observed and a small bowel resection with subtotal colectomy was indicated.

Upon reviewing the literature, this atypical presentation of FMD has yet to be reported. To conclude, our patient was a healthy middle age gentleman who presented with a peculiar case of FMD with pathological involvement of the intimal layer of the terminal branches of the SMA, absence of involvement of the medium sized mesenteric arteries and veins, and unusual lack of renal artery involvement.Unfortunately, the best prognosis of FMD is afforded with early detection and medical management.

\section{References}

1. Touze E, Oppenheim C, Trystram D (2010) Fibromuscular dysplasia of cervical and intracranial arteries. Int J Stroke 5: 296-305. [Crossref]

2. Olin JW, Froehlich J, Gu X (2012) The United States registry for fibromuscular dysplasia: results in the first 447 patients. Circulation 125: 3182-3190. [Crossref] 
3. Kim ESH, Olin JW, Froehlich JB (2013) Clinical manifestations of fibromuscular dysplasia vary by patient sex: a report of the United States registry for fibromuscular dysplasia. J Am Coll Cardiol 62: 2026-2028. [Crossref]
4. Savard S, Steichen O, Azarine A, Azizi M, Jeunemaitre X, et al. (2012) Association between 2 angiographic subtypes of renal artery fibromuscular dysplasia and clinical characteristics. Circulation 126: 3062-3069. [Crossref]

Copyright: $\odot 2018$ Kambiz K. This is an open-access article distributed under the terms of the Creative Commons Attribution License, which permits unrestricted use, distribution, and reproduction in any medium, provided the original author and source are credited. 